REVIEW ARTICLE, SPECIAL ISSUE SPP1236

\title{
Pressure-induced structural transformations in advanced ferroelectrics with relaxor behaviour
}

Boriana Mihailova, ${ }^{\text {a* }}$ Naëmi Waeselmann, ${ }^{\text {a, b }}$ Bernd J. Maier, ${ }^{\text {a, c }}$ Anna-Maria Welsch, ${ }^{\text {a, d }}$ Ross J. Angel, ${ }^{\text {a, e }}$ and Ulrich Bismayer $^{\mathrm{a}}$

${ }^{\mathrm{a}}$ Fachbereich Geowissenschaften, Universität Hamburg, Grindelallee 48, D-20146 Hamburg, Germany;

${ }^{\mathrm{b}}$ Department of Earth and Space Sciences, University of Washington, Seattle, WA 98195-1310, USA;

${ }^{\mathrm{c}}$ Department für Geo- und Umweltwissenschaften, Ludwig-Maximilians Universität München, Theresienstraße 41/II, D80333 München, Germany;

dinstitut für Mineralogie, Leibniz Universität Hannover, Callinstr. 3, D-30167 Hannover, Germany;

e Dipartimento di Geoscienze, Università degli studi di Padova, via G. Gradenigo 6, 35131 Padova, Italy

\begin{abstract}
A series of model perovskite-type relaxor ferroelectrics (pure and A-/B-site doped $\mathrm{PbSc}_{1 / 2} \mathrm{Ta}_{1 / 2} \mathrm{O}_{3}$ and $\mathrm{PbSc}_{1 / 2} \mathrm{Nb}_{1 / 2} \mathrm{O}_{3}$ as well as of $0.9 \mathrm{PbZn}_{1 / 3} \mathrm{Nb}_{2 / 3} \mathrm{O}_{3}-0.1 \mathrm{PbTiO}_{3}$ ) were studied by high-pressure diffraction and inelastic-light scattering in order to elucidate the mesoscopic-scale ferroic atomic arrangements responsible for the superb macroscopic properties of these materials. The combined analysis of the pressure-enhanced phonon anomalies observed by Raman spectroscopy and the pressure-induced long-range order detected by synchrotron X-ray and neutron diffraction revealed that at ambient conditions antiferrodistortive order coexists with the ferroelectric order on the mesoscopic scale. This suggests that the locally polarized spatial nanoregions known as polar nanoregions are ferrielectric in nature and their abundance and mean size depend on both the antiferrodistortive and ferroelectric coupling, which in turn can be tuned by appropriate chemical variations.
\end{abstract}

Keywords: relaxor ferroelectrics, X-ray diffraction, Raman spectroscopy, perovskites, phase transitions

Title Page Footnote:

*Corresponding author. Email: boriana.mihailova@uni-hamburg.de

\section{Introduction}

Relaxor ferroelectrics or relaxors are complex materials with strong intrinsic heterogeneities on the mesoscopic scale, which result in a broad and frequency-dispersive maximum of the dielectric permittivity as a function of temperature (Fig. 1). The strong dielectric, pyroelectric, elastoelectric and optoelectric responses of relaxors over a temperature range near room temperature make them important technological materials used in a number of technological applications such as actuators, capacitors, imaging devices, memories etc. The majority of relaxors with superb properties belong to the perovskite $\left(\mathrm{ABO}_{3}\right)$ structure type (Fig. 2) with $\mathrm{Pb}^{2+}$ occupying the A site and two cations of different valence on the $\mathrm{B}$ site. There are two main groups of 
perovskite-type relaxors, with the general formulae $\mathrm{PbB}^{3+}{ }_{1 / 2} \mathrm{~B}^{5+}{ }_{1 / 2} \mathrm{O}_{3}$ and $\mathrm{PbB}^{2+}{ }_{1 / 3} \mathrm{~B}^{5+}{ }_{2 / 3} \mathrm{O}_{3}$, as well as binary and ternary solid solutions of the type $(1-x) \mathrm{PbB}^{2+}{ }_{1 / 3} \mathrm{~B}^{5+}{ }_{2 / 3} \mathrm{O}_{3}-x \mathrm{PbTiO}_{3}$ and $(1-x)\left[(1-y) \mathrm{PbB}^{3+}{ }_{1 / 2} \mathrm{~B}^{5+}{ }_{1 / 2} \mathrm{O}_{3^{-}}\right.$ $\left.y \mathrm{PbB}^{2+}{ }_{1 / 3} \mathrm{~B}^{5+}{ }_{2 / 3} \mathrm{O}_{3}\right]-x \mathrm{PbTiO}_{3}$ exhibiting a giant piezoelectric effect. A unique feature of perovskite-type relaxors is that their average long-range structure remains cubic well below the temperature of the dielectricpermittivity maximum $\mathrm{T}_{\mathrm{m}}$ or transforms to polar rhombohedral with only a subtle deviation of the rhombohedral unit-cell angle from $90^{\circ}$ (less than $0.15^{\circ}$ near $4 \mathrm{~K}$ ). Yet relaxors have a better performance regarding the macroscopic physical properties than classical ferroelectrics. In fact the relaxor state is characterized by the existence of dynamic nanoregions with coherent polar local structural distortions (see Fig. 3), which flip between different equivalent orientation states [2]. Polar nanoregions nucleate several hundreds of Kelvin above $T_{m}$ at the so-called Burns temperature, $T_{B}$, and on cooling they merge into larger polar nanoregions with slower dynamics at an intermediate characteristic temperature $T^{*}$. Then, below $T_{m}$, they either evolve into long-range ordered ferroelectric domains at the Curie temperature $T_{C}$ or stop flipping at the so-called freezing temperature $\mathrm{T}_{\mathrm{f}}$, without developing long-range ferroelectric order [2-6]. Another intriguing structural property of Pb-based perovskite relaxors is that regardless of the complex chemistry on the $\mathrm{B}$ site, they all exhibit nanoregions with 1:1 chemical order of alternating $\mathrm{B}$ ' and $\mathrm{B}$ " cations along the cubic $\langle 100\rangle$ directions [7,8] as illustrated in Fig. 2. The mesoscopic 1:1 B-site chemical order can be enhanced to long-range order by controlling the rate of crystal growth or by a post-synthesis annealing in the case of $\mathrm{PbB}_{1 / 2} \mathrm{~B}_{1 / 2} \mathrm{O}_{3}[9,10]$, as well as by an appropriate A-site doping in the case of $\mathrm{PbB}_{1 / 3} \mathrm{~B}^{\prime \prime}{ }_{2 / 3} \mathrm{O}_{3}$ [11].

The quest for new environmentally-friendly Pb-free ferroelectrics which are at least as good as Pb-based relaxors calls for further fundamental research on the relationship between chemistry, nanostructure and macroscopic properties. For a long time relaxor behaviour has been mainly attributed to the presence of random local electric fields related to the chemical order/disorder on the B-site [12]. However, temperaturedependent structural studies of A-site doped Pb-based relaxors indicated that the doping-induced local elastic fields as well as disturbance of the system of lone-pair electrons associated with the A-positioned $\mathrm{Pb}^{2+}$ cations have considerably stronger effects on the relaxor state than local electric fields [3,4]. Recent high-pressure structural studies of $\mathrm{PbB}_{1 / 3}^{\prime} \mathrm{B}_{2 / 3} \mathrm{O}_{3}$-type [13-15], $\mathrm{PbB}_{1 / 2}^{\prime} \mathrm{B}_{1 / 2} \mathrm{O}_{3}$-type relaxors [16-28], as well as binary (1$x) \mathrm{PbB}_{1 / 3}^{\prime} \mathrm{B}_{2 / 3} \mathrm{O}_{3}-x \mathrm{PbTiO}_{3}$ solid solutions [29, 30], also emphasized the important role of $\mathrm{Pb}^{2+}$ local order for the relaxor behaviour and indicated that at ambient conditions antiferrodistortive order coexists with the ferroelectric order on the mesoscopic scale [16-29]. This may turn to be the key structural factor for the remarkable relaxor properties. In this paper we present a summary of our high-pressure structural studies (by diffraction and inelastic light scattering) of series of pure and A-/B-site doped $\mathrm{PbSc}_{1 / 2} \mathrm{Ta}_{1 / 2} \mathrm{O}_{3}$ (PST) and $\mathrm{PbSc}_{1 / 2} \mathrm{Nb}_{1 / 2} \mathrm{O}_{3}(\mathrm{PSN})$ samples as well as of $0.9 \mathrm{PbZn}_{1 / 3} \mathrm{Nb}_{2 / 3} \mathrm{O}_{3}-0.1 \mathrm{PbTiO}_{3}(\mathrm{PZN}-0.1 \mathrm{PT})$.

\section{Experimental details}

\subsection{Samples}

We have chosen to simultaneously study PST- and PSN-based systems as model relaxor compounds for several reasons. First, the stoichiometry of the pure compounds matches the mesoscopic 1:1 B-site chemical order typical of all relaxors, which facilitates the synthesis of single crystals with a desired degree of longrange chemical order as well as its detection by X-ray or neutron diffraction. Second, both PST and PSN exhibit a high degree of mesoscopic "ferroicity" at room temperature and ambient pressure, as revealed by the strong X-ray diffuse scattering and anomalous Raman scattering [3,4]; on cooling both PST and PSN develop long-range ferroelectric order but the temperature evolution of the phonon anomalies indicated that there are ferroic species at ambient conditions that are almost insensitive to the temperature decrease, suggesting that 
high pressure may enhance these ferroic species into another type of long-range ferroic order. Third, PST and PSN have the same stoichiometry and tolerance factor $t=\left(r_{i}(A)+r_{i}(O)\right) /\left[\sqrt{2}\left(r_{i}(B)+r_{i}(O)\right)\right], r_{i}$ stands for ionic radius, and differ from each other only by the B"-cation mass and the B"-O interactions, which facilitated the Raman peak assignment for Pb-based relaxors [22, 24, 31] and made possible the detailed analysis of the mesoscopic ferroic atomic arrangements at different temperatures and/or pressures. The identical tolerance factors also mean that the two compounds were expected to exhibit the same pattern of symmetry breaking by the tilting of the octahedra.

In order to analyze the different effects of the chemical substitution on the structure of relaxors we considered the following compositions:

- $\mathrm{PbSc}_{0.5} \mathrm{Ta}_{0.36} \mathrm{Nb}_{0.14} \mathrm{O}_{3}$ (PSTN), which is the case of isovalent substitution of the ferroelectrically active B-site cation $\left(\mathrm{Nb}^{5+}\right.$ for $\left.\mathrm{Ta}^{5+}\right)$, without changing the tolerance factor [22, 26]

- $\mathrm{PbSc}_{0.5} \mathrm{Ta}_{0.36} \mathrm{Nb}_{0.14} \mathrm{O}_{3}$ (PST-Sn), which is the case of B-site aliovalent substitution ( $\mathrm{Sn}^{4+}$ for a pair of $\mathrm{Sc}^{3+}$ and $\mathrm{Ta}^{5+}$ ), without changing the tolerance factor [22, 26]

- $\mathrm{Pb}_{0.78} \mathrm{Ba}_{0.22} \mathrm{Sc}_{0.5} \mathrm{Ta}_{0.5} \mathrm{O}_{3}$ (PST-Ba) and $\mathrm{Pb}_{0.93} \mathrm{Ba}_{0.07} \mathrm{Sc}_{0.5} \mathrm{Nb}_{0.5} \mathrm{O}_{3}$ (PSN-Ba), which is the case of $\mathrm{Pb}^{2+}$ substitution by an isovalent larger-size cation $\left(\mathrm{Ba}^{2+}\right)$ with an isotropic outermost electron shell, i.e. the comparison to pure PST and PSN reveals the effect of chemically induced local elastic stresses as well the disturbance of the system of electronic lone pairs without A-site change imbalance [3, 4, 18, 19, 20, 24, 26]. Since PST-Ba possesses considerably larger domains with long-range chemical B-site order than PST [24], the comparison between PST and PST-Ba allowed us to analyze whether random electric fields associated with the B site or local elastic fields associated to the A site have a stronger impact on the structure.

- $\mathrm{Pb}_{0.98} \mathrm{Bi}_{0.02} \mathrm{Sc}_{0.51} \mathrm{Nb}_{0.49} \mathrm{O}_{3}$ (PSN-Bi), which is the case of $\mathrm{Pb}^{2+}$ substitution by an aliovalent similar-size cation $\left(\mathrm{Bi}^{3+}\right)$ with a stereochemically active lone pair, i.e. the comparison to pure PSN reveals the effect of A-site charge imbalance with no local elastic deformations $[4,19]$.

- $\mathrm{Pb}_{0.66} \mathrm{Sr}_{0.34} \mathrm{Sc}_{0.5} \mathrm{Nb}_{0.5} \mathrm{O}_{3}$ (PSN-Sr), which represents the case of $\mathrm{Pb}^{2+}$ substitution by an isovalent smaller-size cation $\left(\mathrm{Sr}^{2+}\right)$ with an isotropic outermost electron shell [23, 26].

- $\mathrm{Pb}_{0.86} \mathrm{La}_{0.08} \mathrm{Sc}_{0.53} \mathrm{Ta}_{0.47} \mathrm{O}_{2.93}$ (PST-La) and $\mathrm{Pb}_{0.70} \mathrm{La}_{0.23} \mathrm{Sc}_{0.62} \mathrm{Ta}_{0.38} \mathrm{O}_{2.93}$ (PST-La) with corresponding approximate formulae $\mathrm{Pb}_{1-x} \mathrm{La}_{x} \mathrm{Sc}_{(1+x) / 2} \mathrm{Ta}_{(1-x) / 2} \mathrm{O}_{3}, x=0.08$, and $\mathrm{Pb}_{1-x} \mathrm{La}_{x} \mathrm{Sc}_{(1+x) / 2} \mathrm{Nb}_{(1-x) / 2} \mathrm{O}_{3}, x=0.23$, which represents the case of $\mathrm{Pb}^{2+}$ substitution by an aliovalent smaller-size cation $\left(\mathrm{La}^{3+}\right)$ with an isotropic outermost electron shell [23, 26].

- PZN-0.1PT [5, 29], which is on the morphotropic phase boundary, to determine whether or not the main structural features observed in $\mathrm{PbB}_{1 / 2} \mathrm{~B}_{1 / 2} \mathrm{O}_{3}$-type relaxors under high pressure are typical also of relaxor-based systems with rather complex B-site chemistry and a multidomain microstructure.

All samples were synthesized by the high-temperature solution crystal growth method, producing homogeneous single crystals with a linear size varying between $\sim 1-5 \mathrm{~mm}$. The exact chemical composition was determined using electron microprobe (Cameca microbeam SX100 SEM system), by averaging over 100 spatial points. Conventional powder X-ray diffraction (XRD) was used to verify the phase purity as well as to determine the 1:1 B-site chemical long-range order, following the procedure proposed by Stenger and Burggaaf [10]. All studied compounds exhibit no or a low degree $(<0.12)$ of long-range chemical order with a domain mean size ranging between 45 and $4.5 \mathrm{~nm}$ [24]. Plate specimens parallel to one of the cubic $\{100\}$ crystallographic planes were prepared for high-pressure single-crystal experiments. 


\subsection{Diffraction}

\subsubsection{In-house high-precision single-crystal X-ray diffraction}

The equations of state (EoSs) at room temperature up to $9.8 \mathrm{GPa}$ were determined from the pressure dependence of the unit-cell volume $V$ measured with a Huber four-circle single-crystal diffractometer equipped with a sealed-tube source. These experiments were conducted in diamond anvil cells (DACs) using a 4:1 methanol ethanol mixture which is hydrostatic in this pressure range [32]. The unit cell parameters were determined by the method of eight-position diffraction beam centering [33] ensuring a precision in the relative volume $V(p) / V_{0}$ of approximately 0.0001 . The pressure values were calculated from the EoS of a quartz crystal loaded with the sample, with a precision of $\sim 0.01 \mathrm{GPa}$ or better [34].

\subsubsection{Single-crystal $X$-ray diffraction with synchrotron radiation}

Synchrotron single-crystal XRD experiments were performed at the DESY/HASYLAB F1 beamline, using a MarCCD 165 detector. High-pressure experiments at room temperature were conducted in DACs with a radiation wavelength $\lambda=0.5000 \AA$, a sample-to-detector distance of $100 \mathrm{~mm}$, stepwidth of $0.5^{\circ}$ per frame and an exposure time of $120 \mathrm{~s}$. All compounds were measured up to $9.8 \mathrm{GPa}$ in a 4:1 methanol-ethanol mixture. PST, PSN, PST-Nb, PST-Sn, PST-Ba, PST-La, PSN-La, PSN-Sr, and PZN-0.1PT were also measured up to $29 \mathrm{GPa}$ using $\mathrm{He}$ as a pressure-transmitting medium, which within the standard deviation of a ruby pressure marker remains hydrostatic up to $20 \mathrm{GPa}$ and between 20 and $30 \mathrm{GPa}$ exhibits negligible deviation from hydrostaticity [35]. The pressure values were determined from the pressure-induced shift of the R1 photoluminescence line of ruby [36] with an accuracy of $0.1 \mathrm{GPa}$. In the experiments on PST and PSN above $10 \mathrm{GPa}$ the pressure values and hydrostaticity were double checked by following the position and width of the photoluminescence peak of $\mathrm{Sm}^{2+}$-doped $\mathrm{SrB}_{2} \mathrm{O}_{7}$ [37].

\subsubsection{Powder neutron diffraction}

High-pressure time-of-flight neutron powder diffraction of PST, PSN, PST-Ba, and PSN-La was performed at room temperature up to $8 \mathrm{GPa}$ at PEARL, ISIS/RAL. A Paris-Edinburgh pressure cell and a perdeuterated 4:1 methanol-ethanol mixture as a pressure-transmitting medium were used in these experiments. The pressure values were determined using the corresponding EoSs calculated from the $V(p)$-dependencies measured by single-crystal XRD. Rietveld refinements were performed with EXPGUI/GSAS [38, 39], using Bragg peak profiles consisting of a convolution of two back-to-back exponentials with a pseudo-Voigt function and a 12term cosine Fourier series to fit the background.

\subsection{Inelastic light scattering}

\subsubsection{Raman spectroscopy}

High-pressure Raman spectra at room temperature were collected from single-crystal specimens loaded in DAC, using a Horiba Jobin-Yvon T64000 triple-grating spectrometer equipped with an Olympus BH41 microscope and a 50x long-working-distance objective. The measurements were conducted in a backscattering geometry using the 514.5-nm line of an $\mathrm{Ar}^{+}$laser (Coherent 90C FreD). The spectral resolution was $2 \mathrm{~cm}^{-1}$. A 16:3:1 methanol-ethanol-water mixture was used as a pressure transmitting medium up to 10.5 GPa or He up to $29 \mathrm{GPa}$ to ensure hydrostaticity in the corresponding pressure ranges [32, 35]. The pressure was determined by the ruby-line photoluminescence method [36] with an accuracy of $0.1 \mathrm{GPa}$. PST and PSN were also subjected to high-pressure experiments at elevated temperatures using a DAC with a resistance gasket heater, $\mathrm{NaCl}$ as a pressure transmitting medium, and a $\mathrm{Sm}^{2+}$-doped $\mathrm{SrB}_{2} \mathrm{O}_{7}$ as a pressure marker, which 
shows negligible temperature dependence [37]. In all measurements a background spectrum was collected at each pressure and subsequently subtracted from the sample spectrum to eliminate the contribution from the pressure medium. The background-corrected spectra were reduced by the Bose-Einstein phonon occupation factor to eliminate the effect of temperature on the peak intensities and fitted with Lorentzian functions to determine the peak positions, full-widths-at-half-maximum (FWHMs) and intensities.

\subsubsection{Brillouin spectroscopy}

High-pressure experiments were performed on PST at room temperature up to $10 \mathrm{GPa}$. The experiments were carried out in a DAC with the Brillouin spectroscopic system at the German Research Centre for Geosciences (Helmholtz-Zentrum Potsdam Deutsches GeoForschungsZentrum - GFZ), consisting of a Nd:YVO ${ }_{4}$ solid state laser operating at a wavelength of $532 \mathrm{~nm}$ and a Sandercock-type tandem six-pass Fabry-Perot interferometer equipped with a photomultiplier tube. More details are given in Ref. 27.

\section{Results and discussion}

The Raman spectra and XRD patterns of all compounds studied here reveal that pressure induces multistep structural transformations. Measurements on decompression confirmed the reversibility of the structural changes observed by all analytical methods used. Local structural changes occur at two characteristic pressures $p_{1}{ }^{*}$ and $p_{2}{ }^{*}$, which respectively precede two macroscopic phase transitions at critical pressures $p_{\mathrm{c} 1}$ and $p_{\mathrm{c} 2}: p_{1} *<p_{\mathrm{c} 1}<p_{2} *<p_{\mathrm{c} 2}$. As an example, Figs. 4 and 5 show selected data on PST, for which $p_{1} *=1.2$ $\mathrm{GPa}, p_{\mathrm{c} 1}=1.9 \mathrm{GPa}, p_{2} *=3.0 \mathrm{GPa}$, and $p_{\mathrm{c} 2} \sim 5.5$. GPa $[16,20,25]$. At $p_{1} *$ the off-centred displacements of $\mathrm{Pb}^{2+}$ and $\mathrm{B}$-site cations in polar nanoregions decouple, which is revealed by the splitting of the Raman scattering peak near $140 \mathrm{~cm}^{-1}$ (Fig. 4) generated by the $\mathrm{Pb}-\mathrm{BO}_{3}$ translation mode in polar nanoregions [16, 24]. The off-centred B-site cations begin to move to the octahedral centres as revealed by the suppression of the Raman scattering near $240 \mathrm{~cm}^{-1}$ arising the B-cation localized mode in polar nanoregions [16, 24]. The suppression of mesoscopic polar order is also deduced from the weakening of the X-ray diffuse scattering along the cubic $\langle 110\rangle^{*}$ directions arising from polar nanoregions (see Fig. 4) [16, 24]. It should be mentioned that recent studies of the $\langle 110\rangle^{*} \mathrm{X}$-ray diffuse scattering in Pb-based relaxors at ambient conditions [40] revealed that the three-dimensional diffuse-scattering intensity can be best modelled by considering dynamically coupled lattice distortions, without the necessity to introduce static entities of symmetry lower than the symmetry of the paraelectric phase. Our temperature-dependent Raman spectroscopic studies at ambient conditions [3-5, 22, 23] indicate there are uncoupled local structural distortions even above $T_{B}$, as schematically shown in Fig. 3, and we refer to "polar nanoregions" as spatial regions with a certain length of coherence of coupled polar lattice distortions surrounded by a pseudo-paraelectric matrix containing uncoupled local structural distortions. Neutron diffraction analysis (see Fig. 5b) showed that at $p_{1}^{*}$ the offcentred displacements of the $\mathrm{Pb}^{2+}$ cations become locally ordered (with stronger coupling along the cubic $\langle 111\rangle$ directions for PST) and quasi-dynamic long-range antiphase octahedral tilts are developed without breaking the average crystal symmetry [20, 24]. The enhancement of the antiferrodistortive order in the system of $\mathrm{Pb}$ and $\mathrm{O}$ atoms is also mirrored by the kink in the pressure dependence of the position of the Raman peak near $350 \mathrm{~cm}^{-1}$ resulting from $\mathrm{Pb}-\mathrm{O}$ bond stretching the $\mathrm{Pb}-\mathrm{O}$ sheets perpendicular to the cubic body diagonal (see Fig.5c) [16, 24, 25]. The continuous phase transition at $p_{\mathrm{c} 1}$ from a cubic (or pseudocubic) relaxor to a non-polar rhombohedral state is associated with the development of static long-range order of antiphase octahedral tilts ( $a^{-} a^{-} a^{-}$in Glazer notation [41]), resulting in the appearance of odd-odd-odd Bragg peaks (Miller indices in $F m \overline{3} m$ throughout this paper) in reciprocal-space layers reconstructed from singlecrystal synchrotron XRD data (Fig. 4), a discontinuity of the isothermal bulk modulus $K_{\mathrm{T}}$ obtained from $p$ - $V$ 
data (Fig. 5a), and the appearance of a Raman-active low-energy "soft" mode (Fig. 4) [16, 24, 25]. At the second characteristic pressure $p_{2}{ }^{*}$ the octahedral tilts around the three $\langle 100\rangle$ cubic directions become unequal on the mesoscopic scale as deduced from the splitting of the $\mathrm{Pb}-\mathrm{O}$ bond stretching mode near $350 \mathrm{~cm}^{-1}$ (Fig. 5c) $[16,24,25]$ and the transverse acoustic mode experiences strong damping (Fig. 5d) [27]. The second phase transition at $p_{\mathrm{c} 2}$ in PST consists of the development of long-range order of antiparallel $\mathrm{Pb}^{2+}$ displacements and mixed $a^{+} b^{-} b^{-}$tilts is revealed by the appearance of additional sets of even-even-odd Bragg peaks and weaker even-odd-odd Bragg reflections observed by single-crystal synchrotron XRD [25]. The second phase transition is monitored also by the splitting of the Pb-localized mode near $55 \mathrm{~cm}^{-1}$ [25] and the kink in the pressure dependence of the wavenumber $\omega_{\mathrm{SM}}$ of the soft mode [26]. In fact the analysis of $\omega_{\mathrm{SM}}(p)$ reveals that the lowest-energy "soft" mode does not conform to a standard soft-mode behaviour, indicating a significant contribution of order-disorder phenomena to the pressure-induced phase transitions [26]. All of the characteristic and critical pressures established by the pressure evolution of optical phonon modes and X-ray and neutron diffraction are also marked by changes in the pressure evolution of the adiabatic pseudocubic elastic constants calculated from the acoustic phonon modes [27]. In particular, excellent agreement between the pressure behaviour of the adiabatic as well as the isothemal bulk modulus and that of the average phonon energy of the $\mathrm{Pb}-\mathrm{O}$ bond stretching optical mode is found, indicating that the overall crystal compressibility is directly related to the mean local compressibility of the $\mathrm{PbO}_{12}$ cavity [27]. The pressure dependencies of the "soft" mode and the Pb-O bond stretching mode at elevated temperatures show that the first pressure-induced phase transition shifts to lower pressure with temperature increase due to the weakening of the mesoscopic ferroelectric order, while the second pressure-induced phase transition is almost insensitive to temperature changes [28].

PSN exhibits the same sequence of structural transformations as PST but the characteristic and critical pressures are higher and, in addition, the phase-transition pattern at $p_{\mathrm{c} 2}$ is different: no long-range antipolar $\mathrm{Pb}^{2+}$ order develops so the only structural change is a change in long-range tilt order from $a^{-} a^{-} a^{-}$to $a^{-} b^{-} b^{-}$with $0 \leq a<b$ [25]. The solid solution between PST and PSN (PSTN) shows intermediate behaviour with $p_{1}{ }^{*}, p_{\mathrm{c} 1}$, $\mathrm{p}_{2}{ }^{*}$, and $p_{\mathrm{c} 2}$ between the corresponding values of the end members [22, 26]. This indicates that the antiferrodistortive instabilities are enhanced when $\mathrm{Ta}^{5+}$ is replaced by $\mathrm{Nb}^{5+}$. DFT calculations on completely B-site chemically ordered PST and PSN did not reveal any essential difference between the two compounds [25]. Thus we ascribe the difference in the pressure behaviour of PST, PSTN and PSN to the fine-scale difference in the abundance of coherent B'-O-B" linkages (largest for PST, intermediate for PSTN, smallest for PSN), which in turn affect the length of coherence of off-centred $\mathrm{Pb}^{2+}$ displacements coupled along the cubic $\langle 111\rangle$ as revealed by the Raman intensity near $300-350 \mathrm{~cm}^{-1}[22,31]$ and the anisotropic displacement ellipsoids of $\mathrm{Pb}^{2+}$ cations from neutron-diffraction data [22, 24, 25, 31].

The experimental results obtained on the other doped compounds demonstrate the interplay between the chemical disorder on both A and B sites and lattice antiferrodistortive instabilities of perovskite-type relaxors $[24,26]$. The doping-induced renormalizations of the critical pressures suggest that due to the specific chemically-induced local strains heterovalent B-site cation substitution (PST-Sn) as well as homovalent smaller-size A-site cation substitution (PSN-Sr) may stabilize both the antiphase $\mathrm{BO}_{6}$ tilting and the antipolar $\mathrm{Pb}^{2+}$ order, while homovalent larger-size A-site cation substitution (PST-Ba, PSN-Ba) enhances the antiferrodistortive instabilities. Heterovalent smaller-size A-site cation substitution (PST-La, PSN-La) stabilizes the antiphase $\mathrm{BO}_{6}$ tilting but has different effect on the antipolar $\mathrm{Pb}^{2+}$ order, depending on the degree of doping. Interestingly, heterovalent A-site substitution by cations with lone pairs and similar ionic radii also slightly stabilize the antiphase $\mathrm{BO}_{6}$ tilting [19]. It should be emphasized that for all A-site doped 
compounds the effect of local structural distortions prevails over the effect of A- or B-site-related charge imbalance.

Under high pressure PZN-0.1PT also undergoes a phase transition from a relaxor to a rhombohedral state with $a^{-} a^{-} a^{-}$tilts and the pressure/temperature trends of the phonon anomalies also indicate mesoscopic antiferrodistortive order at ambient conditions [5, 29]. In addition, the comparison between the pressure dependence of the structure and properties up to $1 \mathrm{GPa}$ suggests that the remarkable piezoelectric and electromechanical responses in PZN- $x$ PT result from intrinsic ferroic structural inhomogeneities on the mesoscopic scale rather than from the complex multidomain texture [29].

\section{Conclusions}

Combined XRD and Raman spectroscopic analyses when pressure or temperature is varied (see Fig. 6) revealed the coexistence of ferroelectric and antiferrodistrotive mesoscopic order in relaxors at ambient conditions. This implies that the unique relaxor state is caused by the symbiosis of these two types of ferroic order. In other words, the locally polarized spatial nanoregions known as polar nanoregions are ferrielectric rather than ferroelectric in nature. This deduction is based to a great extent on in-situ high-pressure structural studies of relaxors, but is strongly supported by recent acoustic emission [42, 43] and Raman scattering [44] experiments under an external electric field that reveal ferroelectric and antiferroelectric order in the vicinity of $\mathrm{T}_{\mathrm{m}}$ as well as antiferroelectric coupling of $\mathrm{Pb}$ cations and ferroelectric coupling of $\mathrm{B}$-site cations near $\mathrm{T}^{*}$. Chemical variations influence both the ferroelectric and antiferrodistortive order and in such a way may tune the relaxor properties. This study therefore demonstrates the potential of high-pressure structural analysis for elucidating the ambient-pressure nanostructure of advanced functional materials.

Figure Captions:

Figure 1. Temperature dependence of the dielectric permittivity of $\mathrm{PbSc}_{1 / 2} \mathrm{Ta}_{1 / 2} \mathrm{O}_{3}$ at selected frequencies (data from [1]).

Figure 2. Prototypical aristotype structure of perovskite oxides $\left(\mathrm{ABO}_{3}\right)$ : B-site cations form octahedra with the surrounding oxygen anions while A-site cations are placed at the centre of the cavity formed by $8 \mathrm{BO}_{6}$ octahedra. If two types of cations occupy the $\mathrm{B}$ site, 1:1 B-site chemically disordered or ordered domains may exist in the structure having a space group $P m \overline{3} m$ and $F m \overline{3} m$, respectively.

Figure 3. A schematic illustration of the differences between the temperature-driven structural transformations in normal ferroelectrics and relaxor ferroelectrics; polar nanoregions are represented by gray ellipsoids and long-range ordered ferroelectric domains by gray rectangles. If a phase transition occurs in relaxors, the structural deviation from cubic is very small.

Figure 4. Raman scattering of PST and sections of reciprocal-space layers reconstructed from synchrotron XRD data on PST at different pressures [16, 24, 25]; SM in the Raman-scattering indicates the "soft mode". The XRD data at 4.2 GPa were collected from a specimen parallel to one of the cubic $\{110\}$ crystallographic planes and show a systematic absence of pressure-induced $h h h$ Bragg peaks with $h=2 n+1$, indicating the development of antiphase $\mathrm{BO}_{6}$ tilting; the broad weak $1 \overline{1} \overline{1}$ peak originates from chemical 1:1 B-site order alone and is insensitive to pressure increase. The pressure-induced odd-odd-odd XRD peaks are accompanied by X-ray diffuse scattering steaks along the cubic $\langle 100\rangle *$ directions.

Figure 5. (a): Normalized pressure $F=p /\left[3 f(1+3 f)^{5 / 2}\right]$ versus Eulerian strain $f=\left[\left(V_{0} / V\right)^{2 / 3}-1\right] / 2$ calculated from $p$ - $V$ data measured by high-precision single-crystal XRD. (b): Pressure dependence of the tilt angle $\gamma=(180-\angle(\mathrm{B}-\mathrm{O}-\mathrm{B})) / 2$ and the $\mathrm{Pb}$ atomic displacements obtained from neutron diffraction data calculated in $P m \overline{3} m$ (open symbols) or $R \overline{3} c$ (solid symbols). (c): Pressure dependence of the wavenumber of the Raman peak related to Pb-O bond stretching. (d): Pressure dependence of position (gray circles) and the full width at half maximum (FWHM, black circles) of the Brillouin-scattering peak generated from the 
transverse acoustic (TA) phonon mode of PST; the dashed line represents an asymmetric Log-Normal fit to the experimental data of FWHM.

Figure 6. A comparison of the temperature and pressure effects on the Raman spectra of PST. $\bar{Z}(X X) Z$ and $\bar{Z}(X Y) Z$ are the scattering geometries of the spectra measured in air; precise polarized Raman experiments under pressure were hindered by the partial depolarization of light when passing through the diamond anvil. The circles mark the most pronounced spectral changes induced by temperature and pressure. The Raman spectra clearly demonstrate the existence of atomic arrangements with ferroelectric (FE) and antiferrodistortive (AFD) coupling which are correspondingly enhanced by temperature decrease (e.g. the scattering near $240 \mathrm{~cm}^{-1}$ arising from off-centred B-site cations in polar clusters) and pressure increase (e.g. the scattering near 350 $\mathrm{cm}^{-1}$ arising from $\mathrm{Pb}-\mathrm{O}$ bond stretching in ferroic $\mathrm{Pb}-\mathrm{O}$ species). Since $\mathrm{Pb}$ off-centred shifts are considerably larger than those of $\mathrm{B}-$ site cations [25], the data suggest doubling of the perovskite structure with polarity due to ferrielectric order.

\section{Acknowledgements}

Financial support by the Deutsche Forschungsgemeinschaft (SPP 1236, projects MI 1127/2-1 and MI1127/22) is gratefully acknowledged. The authors thank Marin Gospodinov, Bulgarian Academy of Sciences, for synthesizing the single-crystal samples, Carsten Paulmann and William G. Marshall for the excellent support at F1/DESY and Pearl/ISIS, respectively, Jing Zhao, Virginia Tech, for help in high-precision single-crystal XRD data collection, Claude Ederer, Trinity College Dublin, for DFT calculations, and Hauke Marquardt and Sergio Speziale, GFZ Potsdam, for the collaborative Brillouin spectroscopic study. The authors are also grateful to Björn Winkler, Goethe-Universität Frankfurt, for the opportunity to use the gas loader available in his lab.

\section{References}

[1] E. Dul'kin, B. Mihailova, M. Catalan, M. Gospodinov, M. Roth, Phase transformation above $T_{m}$ in $\mathrm{PbSc}_{0.5} \mathrm{Ta}_{0.5} \mathrm{O}_{3}$ relaxor as seen via acoustic emission. Phys. Rev. B 82 (2010), pp 180101(R)/1-4.

[2] A. A. Bokov, Z. G. Ye, Recent progress in relaxor ferroelectrics with perovskite structure. J. Mater. Sci. 41 (2006), pp 31-52.

[3] B. Mihailova, B. J. Maier, C. Paulmann, T. Malcherek, J. Ihringer, M. Gospodinov, R. Stosch, B. Güttler, $\mathrm{U}$. Bismayer, High-temperature structural transformations in relaxor-ferroelectric $\mathrm{PbSc}_{0.5} \mathrm{Ta}_{0.5} \mathrm{O}_{3}$ and $\mathrm{Pb}_{0.78} \mathrm{Ba}_{0.22} \mathrm{Sc}_{0.5} \mathrm{Ta}_{0.5} \mathrm{O}_{3}$. Phys. Rev. B 77 (2008), pp 174106/1-10.

[4] B. J. Maier, B. Mihailova, C. Paulmann, J. Ihringer, M. Gospodinov, R. Stosch, B. Güttler, U. Bismayer, Effect of local elastic strain on the structure of Pb-based relaxors. A comparative study of pure, Ba-and Bidoped $\mathrm{PbSc}_{0.5} \mathrm{Nb}_{0.5} \mathrm{O}_{3}$. Phys. Rev. B 79 (2009), pp 224108/1-8.

[5] N. Waeselmann, B. Mihailova, B. J. Maier, C. Paulmann, M. Gospodinov, V. Marinova, U. Bismayer, Local structural phenomena in pure and $\mathrm{Ru}$-doped $0.9 \mathrm{PbZn}_{1 / 3} \mathrm{Nb}_{2 / 3} \mathrm{O}_{3}-0.1 \mathrm{PbTiO}_{3}$ near the morphotropic phase boundary as revealed by Raman spectroscopy, Phys. Rev. B 83 (2011), pp 214104/1-13.

[6] E. Dul'kin, M. Roth, P. E. Janolin, B. Dkhil, Acoustic emission study of phase transitions and polar nanoregions in relaxor-based systems: Application to the $\mathrm{PbZn}_{1 / 3} \mathrm{Nb}_{2 / 3} \mathrm{O}_{3}$ family of single crystals, Phys. Rev. B 73 (2006), pp 012102/1-4.

[7] Y. Yan, S. J. Pennycook, Z. Xu, and D. Viehland, Determination of the ordered structures of $\mathrm{Pb}\left(\mathrm{Mg}_{1 / 3} \mathrm{Nb}_{2 / 3}\right) \mathrm{O}_{3}$ and $\mathrm{Ba}\left(\mathrm{Mg}_{1 / 3} \mathrm{Nb}_{2 / 3}\right) \mathrm{O}_{3}$ by atomic-resolution Z-contrast imaging. Appl. Phys. Lett. 72 (1998), pp 3145-3147. 
[8] A. I. Frenkel, D. M. Pease, J. Giniewicz, E. A. Stern, D. L. Brewe, M. Daniel, J. Budnick, Concentrationdependent short-range order in the relaxor ferroelectric (1-x) Pb(Sc, $\mathrm{Ta}) \mathrm{O}_{3}-x \mathrm{PbTiO}_{3}$, Phys. Rev. $\mathrm{B} 70$ (2004), pp 014106/1-12.

[9] B. Mihailova, M. Gospodinov, B. Güttler, D. Petrova, R. Stosch, U. Bismayer. Ferroic nanoclusters in relaxors: the effect of oxygen vacancies. J. Phys.: Condens. Matter 19 (2007), pp 246220/1-10.

[10] C. G. F. Stenger and A. J. Burggaaf, Order-disorder reactions in the ferroelectric perovskites $\mathrm{Pb}\left(\mathrm{Sc}_{1 / 2} \mathrm{Nb}_{1 / 2}\right) \mathrm{O}_{3}$ and $\mathrm{Pb}\left(\mathrm{Sc}_{1 / 2} \mathrm{Ta}_{1 / 2}\right) \mathrm{O}_{3}$.I. Kinetics of the ordering process. Phys. Stat. Sol. (a) 61 (1980), pp 275-285.

[11] X. Long, A. A. Bokov, Z.-G. Ye, W. Qu, X. Tan, Enhanced ordered structure and relaxor behaviour of $0.98 \mathrm{~Pb}\left(\mathrm{Mg}_{1 / 3} \mathrm{Nb}_{2 / 3}\right) \mathrm{O}_{3}-0.02 \mathrm{La}\left(\mathrm{Mg}_{2 / 3} \mathrm{Nb}_{1 / 3}\right) \mathrm{O}_{3}$ single crystals. J. Phys.: Condens. Matter 20 (2008), pp 015210/1-7.

[12] R. A. Cowley, S. N. Gvasaliya, S. G. Lushnikov, B. Roessli, G. M. Rotaru, Relaxing with relaxors: a review of relaxor ferroelectrics. Adv. Phys. 60 (2011), pp 229-327.

[13] B. Chaabane, J. Kreisel, B. Dkhil, P. Bouvier, M. Mezouar, Pressure-induced suppression of the diffuse scattering in the model relaxor ferroelectric $\mathrm{PbMg}_{1 / 3} \mathrm{Nb}_{2 / 3} \mathrm{O}_{3}$. Phys. Rev. Lett. 90 (2003), pp 257601/1-4.

[14] P. E. Janolin, B. Dkhil, P. Bouvier, J. Kreisel, P. A. Thomas, Pressure instabilities up to 46 GPa in the relaxor ferroelectric $\mathrm{PbZn}_{1 / 3} \mathrm{Nb}_{2 / 3} \mathrm{O}_{3}$. Phys. Rev. B 73 (2006), pp 094128-xx.

[15] G.-M. Rotaru, S. N. Gvasaliya, V. Pomjakushin, B. Roessli, Th. Strässle, S. G. Lushnikov, T. A. Shaplygina, P. Günter, Atomic displacements in $\mathrm{PbMg}_{1 / 3} \mathrm{Nb}_{2 / 3} \mathrm{O}_{3}$ under high pressures. J. Phys.: Condens. Matter 20 (2008), pp 104235/1-4.

[16] B. Mihailova, R. J. Angel, A.-M. Welsch, J. Zhao, J. Engel, C. Paulmann, M. Gospodinov, H. Ahsbahs, R. Stosch, B. Güttler, U. Bismayer, Pressure-induced phase transition in $\mathrm{PbSc}_{0.5} \mathrm{Ta}_{0.5} \mathrm{O}_{3}$ as a model Pb-based perovksite-type relaxor ferroelectric. Phys. Rev. Lett. 101 (2008), pp 017602/1-4.

[17] A.-M. Welsch, B. Mihailova, R. Stosch, B. Güttler, M. Gospodinov, U. Bismayer, High-pressure Raman spectroscopic study on the relaxor ferroelectric $\mathrm{PbSc}_{0.5} \mathrm{Nb}_{0.5} \mathrm{O}_{3}$. J. Phys.: Condens. Matter 21 (2009), pp 235901/1-6.

[18] A.-M. Welsch, B. J. Maier, J. M. Engel, B. Mihailova, R. J. Angel, C. Paulmann, M. Gospodinov, A. Friedrich, R. Stosch, B. Güttler, D. Petrova, U. Bismayer, Effect of Ba-incorporation on pressure-induced structural changes in the relaxor ferroelectric PbSc${ }_{0.5} T_{0.5} \mathrm{O}_{3}$. Phys. Rev. B 80 (2009), pp 104118/1-7.

[19] B. J. Maier, A.-M. Welsch, R. J. Angel, B. Mihailova, J. Zhao, J. M. Engel, L. A. Schmitt, C. Paulmann, M. Gospodinov, A. Friedrich, U. Bismayer, A-site doping induced renormalization phenomena in $\mathrm{PbSc}_{0.5} \mathrm{Nb}_{0.5} \mathrm{O}_{3}$ under high pressure. Phys. Rev. B 81 (2010), pp 174116/1-8.

[20] B. J. Maier, R. J. Angel, W. G. Marshall, B. Mihailova, C. Paulmann, J. M. Engel, M. Gospodinov, A.M. Welsch, D. Petrova, U. Bismayer, Octahedral tilting in Pb-based relaxor ferroelectrics at high pressure. Acta Cryst. B 66 (2010), pp 280-291.

[21] B. J. Maier, R. J. Angel, B. Mihailova, W. G. Marshall, M. Gospodinov, U. Bismayer, High-pressure powder neutron diffraction study on lead scandium niobate. J. Phys.: Condens. Matter 23 (2011), pp 035902/1-5.

[22] A.-M. Welsch, B. J. Maier, B. Mihailova, R. J. Angel, J. Zhao, C. Paulmann, J. M. Engel, M. Gospodinov, V. Marinova, U. Bismayer, Transformation processes in relaxor ferroelectric $\mathrm{PbSc}_{0.5} \mathrm{Ta}_{0.5} \mathrm{O}_{3}$ heavily doped with Nb and Sn. Z. Kristallogr. 226 (2011), pp 126-137.

[23] B. J. Maier, A.-M. Welsch, B. Mihailova, R. J. Angel, J. Zhao, C. Paulmann, J. M. Engel, W. G. Marshall, M. Gospodinov, D. Petrova, U. Bismayer, Effect of La doping on the ferroic order in Pb-based relaxor ferroelectrics. Phys. Rev. B 83 (2011), pp 134106/1-12. 
[24] B. Mihailova, R.J. Angel, B. J. Maier, A.-M. Welsch, J. Zhao, M. Gospodinov, U. Bismayer, The structural state of lead-based relaxor ferroelectrics under pressure. IEEE Transactions on Ultrasonics, Ferroelectrics and Frequency Control, 58 (2011), pp 1905 - 1913.

[25] B. J. Maier, N. Waeselmann, B. Mihailova, R. J. Angel, C. Ederer, C. Paulmann, M. Gospodinov, A. Friedrich, U. Bismayer, The structural state of relaxor ferroelectrics $\mathrm{PbSc}_{0.5} \mathrm{Ta}_{0.5} \mathrm{O}_{3}$ and $\mathrm{PbSc}_{0.5} \mathrm{Nb}_{0.5} \mathrm{O}_{3}$ at high pressures up to 30 GPa, Phys. Rev. B, 84 (2011), pp 174104/1-11.

[26] B. Mihailova, N. Waeselmann, B. J. Maier, R. J. Angel, T. Prüßmann, C. Paulmann, M. Gospodinov, U. Bismayer, Chemically-induced renormalization phenomena in Pb-based relaxor ferroelectrics under high pressure, J. Phys.: Cond. Matter. 25, 115403.

[27] H. Marquardt, N. Waeselmann, M. Wehber, R. J. Angel, M. Gospodinov, B. Mihailova, High-pressure Brillouin sctattering of single-cystal $\mathrm{PbSc}_{0.5} \mathrm{Ta}_{0.5} \mathrm{O}_{3}$ relaxor ferroelectric, Phys. $\mathrm{Rev}$. $\mathrm{B}$, under revision. [28] N. Waeselmann, B. Mihailova, M. Gospodinov, U. Bismayer, In-situ high-temperature high-pressure Raman spectroscopy on lead-based relaxor ferroelectrics $\mathrm{PbSc}_{1 / 2} \mathrm{Nb}_{1 / 2} \mathrm{O}_{3}$ and $\mathrm{PbSc}_{1 / 2} \mathrm{Ta}_{1 / 2} \mathrm{O}_{3}$, J. Phys.: Cond. Matter. 25, 155902.

[29] N. Waeselmann, B. J. Maier, B. Mihailova, R. J. Angel, J. Zhao, M. Gospodinov, C. Paulmann, N. Ross, $\mathrm{U}$. Bismayer, Pressure induced structural transformations in pure and Ru-doped $0.9 \mathrm{PbZn}_{1 / 3} \mathrm{Nb}_{2 / 3} \mathrm{O}_{3^{-}}$ 0.1PbTiO 3 near the morphotropic phase boundary, Phys. Rev. B 85 (2012), pp 014106/1-10.

[30] B. Chaabane, J. Kreisel, P. Bouvier, G. Lucazeau, B. Dkhil, Effect of high pressure on the Pb.Mg1/3Nb2/3.O3-PbTiO3 solid solution: A Raman scattering investigation. Phys. Rev. B 70 (2004), pp $134114 / 1-5$

[31] B. Mihailova, U. Bismayer, B. Güttler, M. Gospodinov, L. Konstantinov, Local structure and dynamics in relaxor-ferroelectric $\mathrm{PbSc}_{1 / 2} \mathrm{Nb}_{1 / 2} \mathrm{O}_{3}$ and $\mathrm{PbSc}_{1 / 2} \mathrm{Ta}_{1 / 2} \mathrm{O}_{3}$ single crystals. J. Phys.: Condens. Matter 14 (2002), 1091-1105.

[32] R. J. Angel, M. Bujak, J. Zhao, G. D. Gatta, S. D. Jacobsen, Effective hydrostatic limits of pressure media for high-pressure crystallographic studies. J. Appl. Cryst. 40 (2007), pp 26-32.

[33] R. J. Angel, L. W. Finger, SINGLE: a program to control single-crystal diffractometers. J. Appl. Cryst. 44 (2011), pp 247-251.

[34] R. J. Angel, D. R. Allan, R. Miletich, W. L. Finger, The use of quartz as an internal pressure standard in high-pressure crystallography. J. Appl. Cryst. 30, 461 (1997), pp 461-466.

[35] S. Klotz, J.-C. Chervin, P. Munsch, G. Le Marchand, Hydrostatic limits of 11 pressure transmitting media. J. Phys. D: Appl. Phys. 42 (2009), pp 075413/1-7.

[36] R. G. Munro, G. J. Piermarini, S. Block, W. B. Holzapfel, Model line-shape analysis for the ruby R lines used for pressure measurements. J. Appl. Phys. 57 (1985), pp 165-169.

[37] F. Datchi, A. Dewaele, P. Loubeyre, R. LeToullec, Y. Le Godec, B. Canny, Optical pressure sensors for high-pressure-high-temperature studies in a diamond anvil cell. High Press. Res. 27 (2007), pp 447-463.

[38] B. H. Toby, EXPGUI, a graphical user interface for GSAS. J. Appl. Cryst. 34 (2001), pp 210-213. [39] A. C. Larson, R. B. von Dreele, General Structure Analysis System (GSAS). Los Alamos National Laboratory Report LAUR (2004), pp 86-748.

[40] A. Bosak, D. Chernyshov, S. Vakhrushev, M. Krisch. Diffuse scattering in relaxor ferroelectrics: true three-dimensional mapping, experimental artefacts and modelling. Arcta Cryst. B 28 (2011), pp 117-123. [41] A. M. Glazer, The classification of tilted octahedra in perovskites. Arcta Cryst. B 28 (1972), pp 33843392.

[42] E. Dul'kin, E. Mojaev, M. Roth, I. P. Raevski, S. A. Prosandeev, Nature of thermally stimulated acoustic emission from $\mathrm{PbMg}_{1 / 3} \mathrm{Nb}_{2 / 3} \mathrm{O}_{3}-\mathrm{PbTiO}_{3}$ solid solutions. Appl. Phys. Lett. 94 (2009), pp 252904/1-3. 
[43] E. Dul'kin, B. Mihailova, M. Gospodinov, M. Roth, Electric field dependence of the characteristic temperatures in $\mathrm{PbSc}_{0.5} \mathrm{Ta}_{0.5} \mathrm{O}_{3}$ and $\mathrm{Pb}_{0.78} \mathrm{Ba}_{0.22} \mathrm{Sc}_{0.5} \mathrm{Ta}_{0.5} \mathrm{O}_{3}$ relaxors studied via acoustic emission. Eur. Phys. Lett. 94 (2011), 57002/1-5.

[44] B. J. Maier, T. Steilmann, M. Gospodinov, U. Bismayer, B. Mihailova, Influence of electric field on local phase transformations in relaxor ferroelectrics $\mathrm{PbSc}_{0.5} \mathrm{Ta}_{0.5} \mathrm{O}_{3}$ and $\mathrm{Pb}_{0.78} \mathrm{Ba}_{0.22} \mathrm{Sc}_{0.5} \mathrm{Ta}_{0.5} \mathrm{O}_{3}$. J. Appl. Phys. 112 (2012), pp 124111/1-6. 




Figure 1 (colour online) 
- A-site cation

- B-site cation

- B' $\bigcirc$ B"

- Oxygen
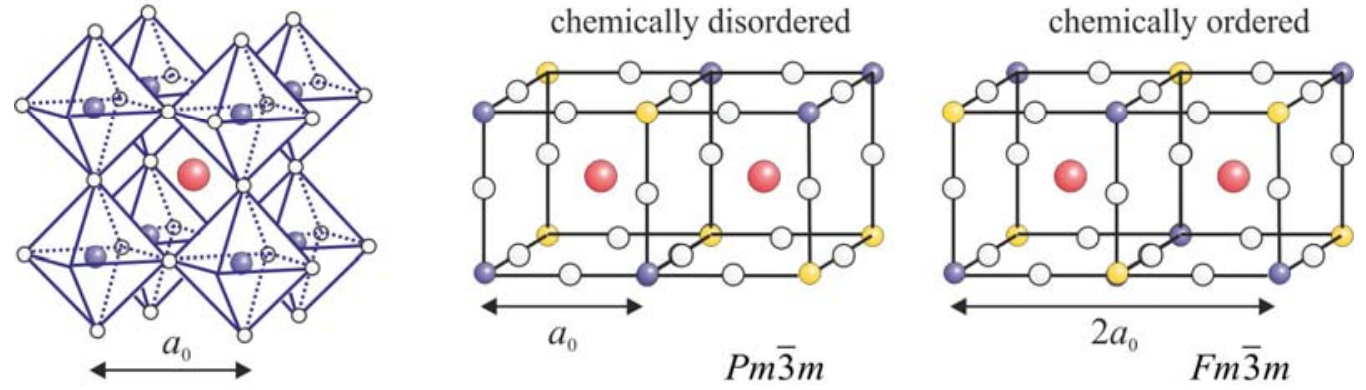

Figure 2 (colour online) 


\section{Normal ferroelectrics}

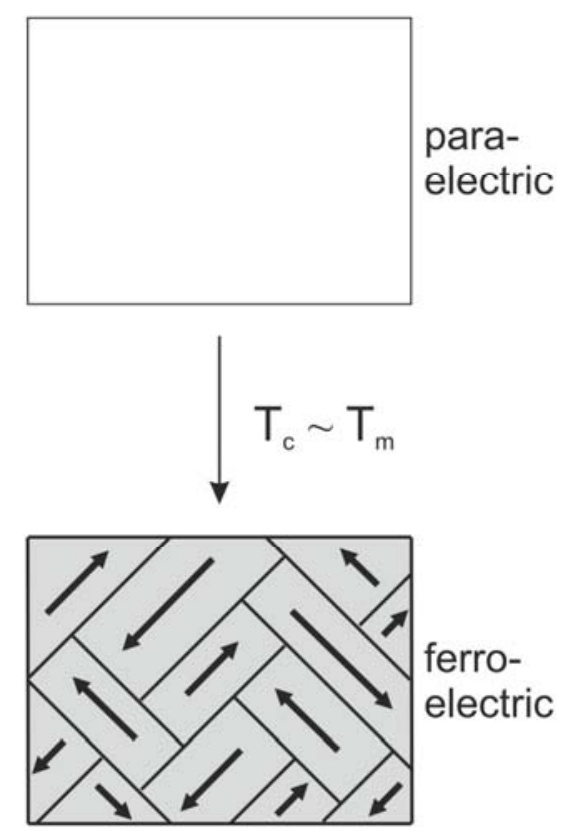

\section{Relaxor ferroelectrics}

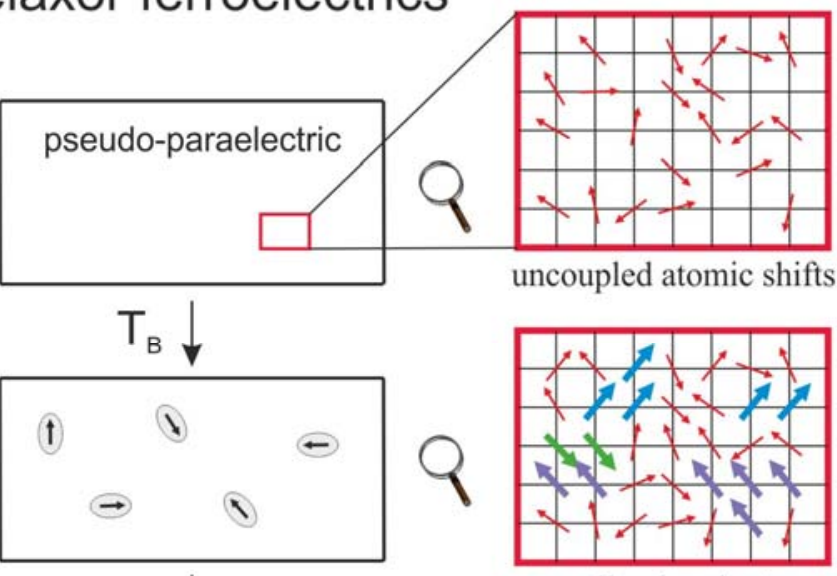

small polar clusters
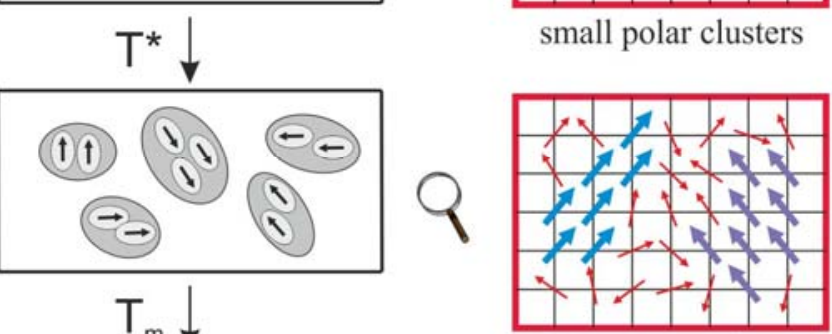

larger polar clusters long-range ordered ferroelectric domains

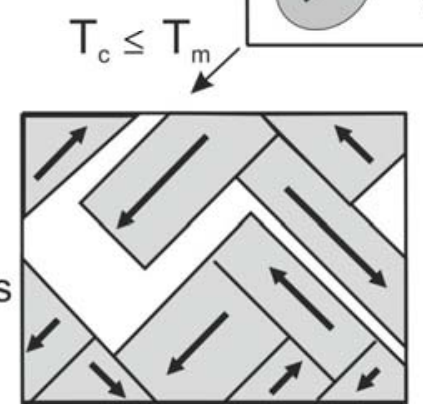

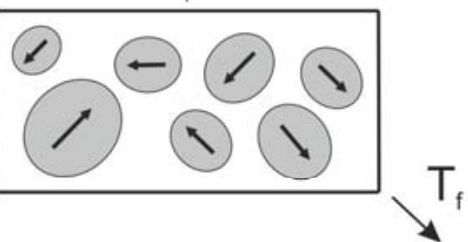

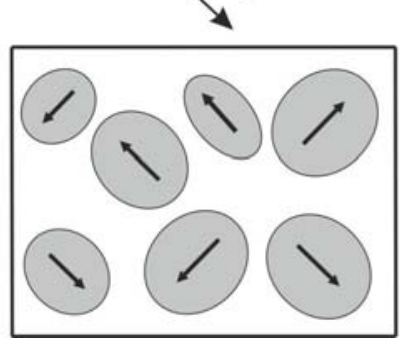

static polar nanoregions

Figure 3 (colour online) 

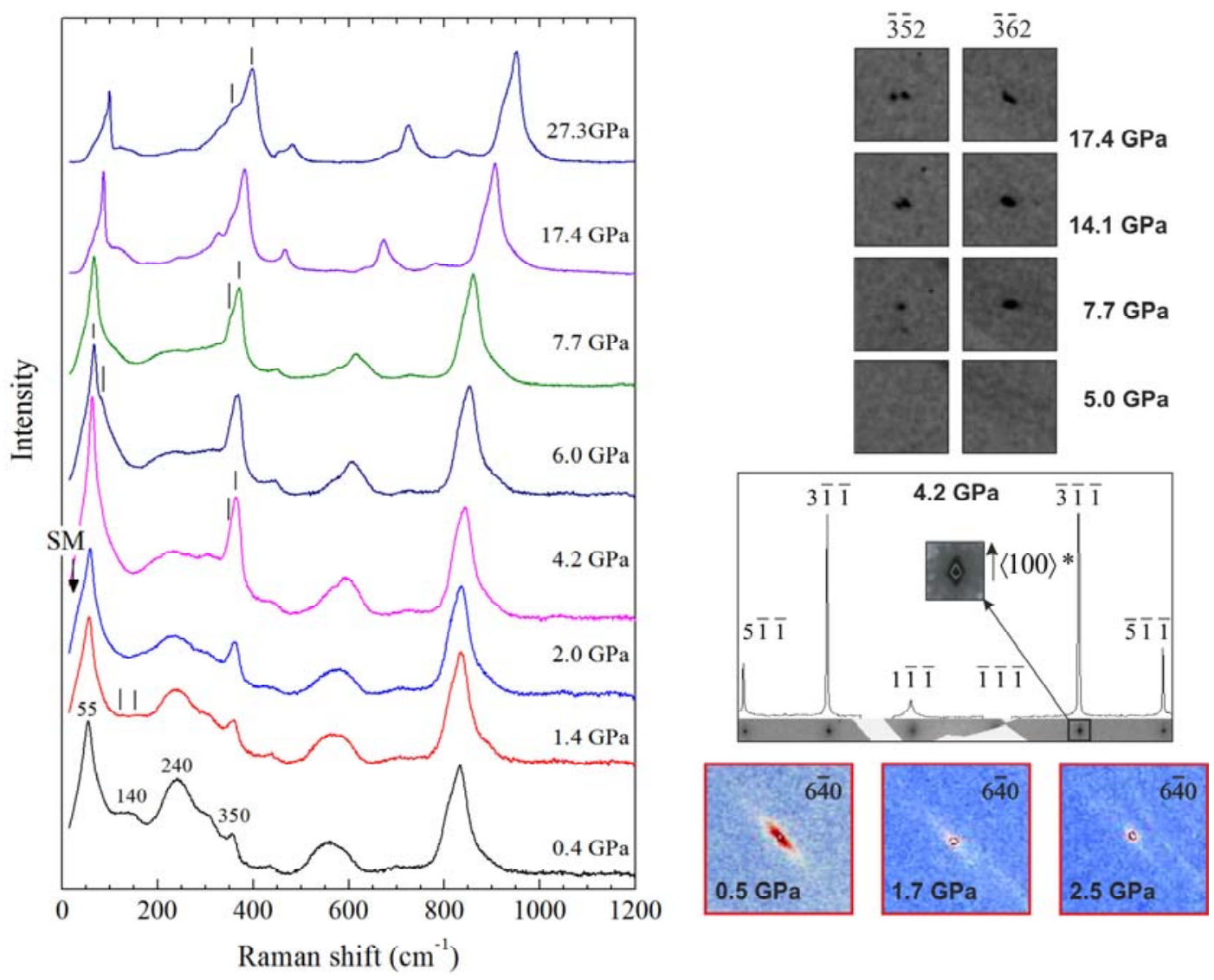

Figure 4 (colour online) 

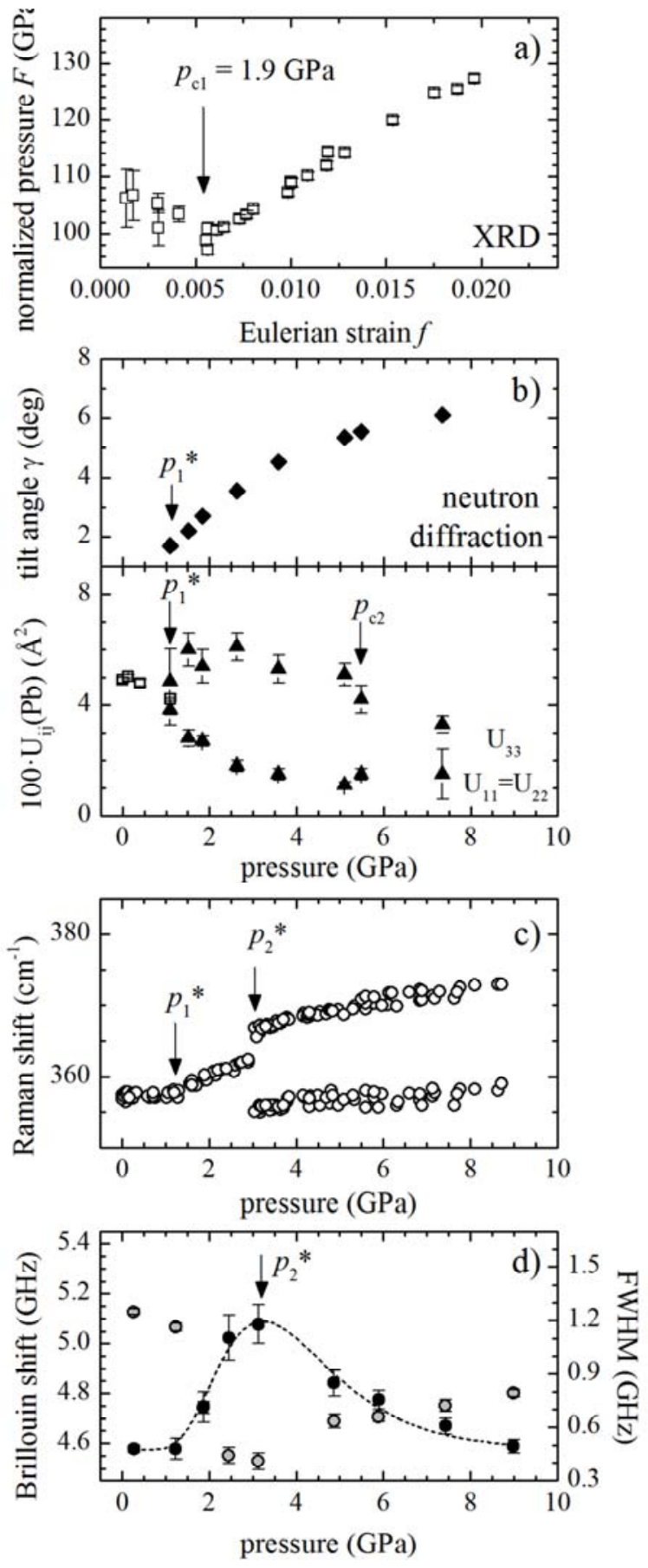

Figure 5 

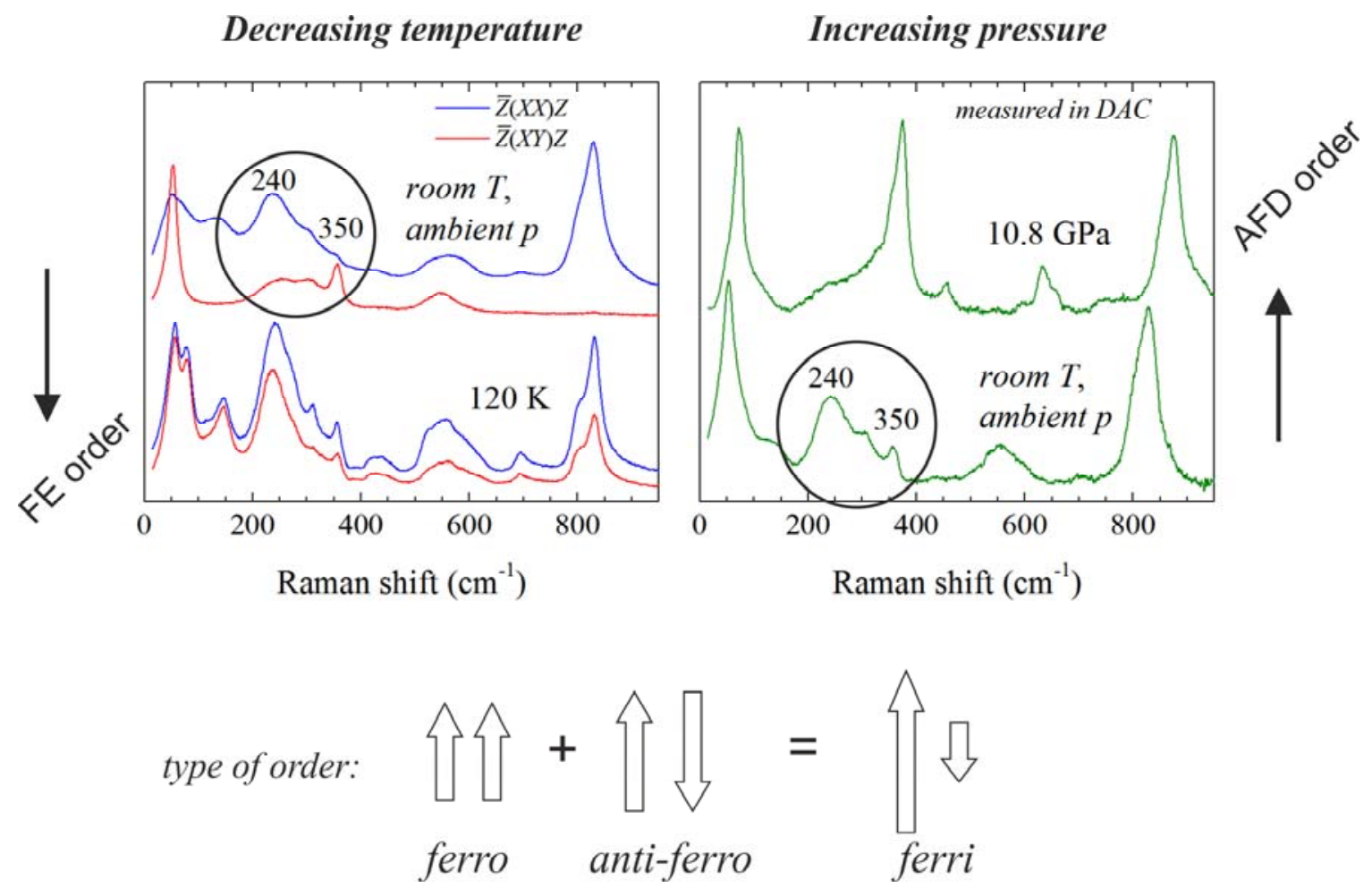

Figure 6 (colour online) 